\title{
Lipocalin-2/Neutrophil Gelatinase-B Associated Lipocalin Is Strongly Induced in Hearts of Rats With Autoimmune Myocarditis and in Human Myocarditis
}

\author{
Limin Ding, MD; Haruo Hanawa, MD; Yoshimi Ota, $\mathrm{PhD}^{\dagger}$; Go Hasegawa, MD*; \\ Kazuhisa Hao, MD; Fuyuki Asami, MD**; Ritsuo Watanabe, MD; \\ Tsuyoshi Yoshida, MD; Ken Toba, MD; Kaori Yoshida, BS; Minako Ogura, BS; \\ Makoto Kodama, MD; Yoshifusa Aizawa, MD
}

\begin{abstract}
Background: Lipocalin-2/neutrophil gelatinase-B associated lipocalin (Lcn2/NGAL) is involved in the transport of iron and seems to play an important role in inflammation. A recent study has reported that it is also expressed in the failing heart and may be a biomarker not only for renal failure but also for heart failure. Because Lcn2/NGAL is thought to be induced by interleukin-1, it might be strongly induced in the presence of myocarditis.

Methods and Results: This study investigated the expression of Lcn2/NGAL in rat experimental autoimmune myocarditis (EAM) and in human myocarditis. In EAM hearts, the expression of Lcn2/NGAL was markedly increased (>100-fold at an early stage), and in human myocarditis it was also highly expressed compared with non-inflammatory failing hearts. Lcn2/NGAL expressing cells in hearts with EAM and human myocarditis were identified as cardiomyocytes, vascular wall cells, fibroblasts and neutrophils. Lcn2/NGAL in EAM rats was also expressed in the liver. Plasma Lcn2/NGAL levels abruptly increased at an early stage of EAM, and high levels were initially sustained during the inflammatory stage, then decreased with recovery. In contrast, levels of B-type natriuretic peptide increased only slowly as the disease progressed.
\end{abstract}

Conclusions: Cardiomyocytes, vascular wall cells and fibroblasts in myocarditis strongly express Lcn2/NGAL via proinflammatory cytokines. (Circ $J$ 2010; 74: 523-530)

Key Words: Biomarker; Cardiomyopathy; Cytokine; Myocarditis; Reactive oxygen species

$\mathbf{L}$ ipocalin-2/neutrophil gelatinase-B associated lipocalin ( $\mathrm{Lcn} 2 / \mathrm{NGAL})$ is a protein associated with neutrophil gelatinase, ${ }^{1}$ and plays a role as an antibacterial factor. ${ }^{2}$ It is suggested that Lcn2/NGAL may play other important roles in inflammation because it is involved in the transport of iron across cell membranes, ${ }^{3}$ and is thought also to closely regulate apoptosis. ${ }^{4}$ Other recent reports have suggested that Lcn2/NGAL has a protective effect against oxidant stress. ${ }^{5}$

Lcn2/NGAL was initially identified in activated neutrophils, then subsequently shown that many other types of cells, including kidney tubular cells, lung type II alveolar epithelial cells, and liver hepatocytes may produce NGAL in response to injuries. ${ }^{6,7}$ Recent investigations of Lcn2/NGAL in renal disease suggest that it may be a promising new biomarker for acute renal failure. It has been speculated that the increase in the Lcn2/NGAL level after renal tubular injury may serve to limit injury in recurrent insults or even ameliorate the degree of damage in an ongoing insult. ${ }^{7}$

Numerous studies have previously reported that Lcn2/ NGAL is a biomarker of renal failure associated with heart diseases. ${ }^{8-11}$ Those studies demonstrated that urine and serum concentrations of Lcn2/NGAL, which was produced by the proximal tubules, were increased in patients with acute renal failure after cardiac surgery or in chronic heart failure. On the other hand, a few studies have reported that Lcn2/NGAL is expressed also in the heart. ${ }^{12,13} \mathrm{Lcn} 2 / \mathrm{NGAL}$ was highly expressed in transplanted hearts ${ }^{12}$ or following X-ray exposure, ${ }^{13}$ and Lcn2/NGAL expressing cells were found to be heart granulocytes. ${ }^{12}$ In addition, a recent study has demonstrated that Lcn $2 / \mathrm{NGAL}$ is produced by cardiomyocytes and resident cells in failing hearts, and might serve as a useful biomarker for heart failure. ${ }^{14}$ Our previous study using micro-

Received July 3, 2009; revised manuscript received October 28, 2009; accepted October 29, 2009; released online January 7, 2010 Time for primary review: 12 days

Division of Cardiology, *Division of Cellular and Molecular Pathology, **Department of Thoracic and Cardiovascular Surgery, Niigata University Graduate School of Medical and Dental Sciences and Department of Medical Technology, School of Health Sciences, Faculty of Medicine, Niigata University, Niigata, Japan

Mailing address: Haruo Hanawa, MD, Division of Cardiology, Niigata University Graduate School of Medical and Dental Sciences, 1757 Asahimachi-dori, Niigata 951-8120, Japan

ISSN-1346-9843 doi:10.1253/circj.CJ-09-0485

All rights are reserved to the Japanese Circulation Society. For permissions, please e-mail: cj@j-circ.or.jp 
array analysis in cardiomyocytes also demonstrated that Lcn2/ NGAL was a protein that was strongly induced in cardiomyocytes purified from rats with experimental autoimmune myocarditis (EAM). ${ }^{15}$ It is known also that interleukin-1 (IL-1) plays an important role ${ }^{16,17}$ and is highly expressed in hearts with myocarditis, ${ }^{18,19}$ and because IL-1 induces Lcn2/ NGAL expression, ${ }^{6}, 14$ we surmised that the Lcn2/NGAL mRNA level might also be increased in hearts with myocarditis. Accordingly, we investigated the expression of Lcn2/ NGAL in rat EAM and in human myocarditis.

\section{Methods}

\section{Animals}

Male Lewis rats were obtained from Charles River, Japan (Atsugi, Kanagawa, Japan) and were maintained in our animal facilities until they reached 8 weeks of age in preparation for the EAM experiments. Throughout the studies, all animal experiments in our institute followed the guidelines for the care and use of laboratory animals published by the US National Institutes of Health.

\section{Induction of EAM}

Cardiac myosin was prepared from the ventricular muscle of porcine hearts as previously described. ${ }^{20}$ To produce EAM each rat was immunized on day 0 with $0.2 \mathrm{ml}$ of an emulsion containing porcine cardiac myosin, along with an equal volume of complete Freund's adjuvant supplemented with $10 \mathrm{mg} / \mathrm{ml}$ of Mycobacterium tuberculosis H37RA (Difco, Detroit, MI, USA).

\section{Purification of Cells From EAM Hearts}

EAM rats were killed on day 18 . For analysis of Lcn2/NGAL and its specific receptor $(24 \mathrm{p} 3 \mathrm{R})$ gene expressing cells, fractions of cardiomyocytes $(n=5), \alpha \beta$ T cells $(n=5), C D 11 b^{+}$ cells $(n=5)$ and non-cardiomyocytic non-inflammatory (NCNI) cells (mainly fibroblasts, smooth muscle cells, and endothelial cells) $(n=6)$ were isolated and purified as previously described. ${ }^{20}$ Cells from both ventricles were isolated by collagenase perfusion treatment for $20 \mathrm{~min}$ using a Langendorff apparatus. The isolated cells were serially separated through $38-\mu \mathrm{m}$ stainless steel sieves to yield cardiomyocytes, and then through $20-\mu \mathrm{m}$ sieves to yield noncardiomyocytic (NC) cells. Because almost all inflammatory cells in EAM are $\alpha \beta$ T cells and CD11 bc ${ }^{+}$cells, the NC cells were further separated into $\alpha \beta \mathrm{T}$ cells, CD11 bc cells and NCNI cells, such as fibroblasts, smooth muscle cells or endothelial cells, with anti-PE micro beads (Miltenyi Biotec, Bergisch Gladbach, Germany) and a MACS magnetic cell sorting system (Miltenyi Biotec). For this process appropriate monoclonal antibodies, namely PE-conjugated TCR $\alpha \beta$ (R73) and CD11bc (OX-42) (Pharmingen, San Diego, CA, USA), were used.

\section{RNA Extraction and Real-Time Reverse Transcriptase- Polymerase Chain Reaction (RT-PCR)}

To examine the time course of Lcn $2 /$ NGAL and IL- $1 \beta$ expression in hearts, a group of 8 -week old normal rats $(n=3)$ was killed, and their results compared with EAM rats killed on days $9,12,15,18,30(n=3$ for each $)$ and $60(n=4)$. In each case a small part of the cardiac ventricle was extracted. A separate group of EAM rats was killed on day 13, and in each a small part of the cardiac ventricle, liver, spleen and kidney ( $\mathrm{n}=4$ in each group) was extracted to examine local Lcn2/ NGAL expression in comparison with 8-week old normal rats and control rats injected with adjuvant alone. Total RNA was isolated from the materials described above using Trizol (Invitrogen, Tokyo, Japan). Synthesis of cDNA was carried out using $2-5 \mu \mathrm{g}$ of total RNA with random primers and murine Moloney leukemia virus reverse transcriptase. To create the plasmids used for the standard, rat Lcn2/NGAL, rat $24 \mathrm{p} 3 \mathrm{R}$, rat IL- $1 \beta$, and human Lcn $2 / \mathrm{NGAL}$ mRNA were amplified from EAM heart or myocarditis autopsy specimenderived cDNA using the primer pairs (rat-Lcn2/NGAL, sense primer 5'-gactcaactcagaacttgatccct-3' and antisense primer 5'-agctctgtatctgagggtagctgt-3'; rat-24p3R, sense primer 5'-ctaccgaaacatctggaaaaatct-3' and antisense primer s'-gtactgaggatagcagaagcttgg-3'; human-Lcn2/NGAL, sense primer 5'-tgagctgaaagaagacaagagcta-3' and antisense primer 5'-gatgaagttctcctttagttccga-3') and the primers as reported previously. ${ }^{16}$ PCR-amplified cDNA inserts were directly inserted into the pGEM-T easy vector, and recombinant plasmids were isolated following transformation into Escherichia coli JM109-competent cells using a MagExtractor plasmid kit (Toyobo, Osaka, Japan). Absolute copy numbers of their mRNA were also measured by quantitative real-time RT-PCR using a LightCycler instrument (Roche Diagnostics, Tokyo, Japan) together with the same primers and SYBR Premix Ex Taq (Takara, Otsu, Japan). After an initial denaturation step of $10 \mathrm{~min}$ at $95^{\circ} \mathrm{C}$, a 3 -step cycling procedure (denaturation at $95^{\circ} \mathrm{C}$ for $10 \mathrm{~s}$, annealing at $62^{\circ} \mathrm{C}$ for $10 \mathrm{~s}$, and extension at $72^{\circ} \mathrm{C}$ for $13 \mathrm{~s}$ ) was used for 45 cycles. The absolute copy numbers of particular transcripts were calculated by LightCycler software using a standard curve approach.

\section{Method for ELISA}

To examine the time course of plasma concentrations of Lcn2/NGAL, IL-1 $\beta$, and B-type natriuretic peptide (BNP), blood samples were obtained from the tail vein of 8-week old normal rats, and from control rats injected with adjuvant alone and EAM rats on days 9, 13, 17, 21, 25, 29, 33, 45 and $60(\mathrm{n}=4$ for each). For the rats, plasma Lcn2/NGAL concentrations were determined with a Rat NGAL ELISA kit according to the manufacturer's instructions (BioPorto Diagnostics, Gentofte, Denmark), BNP concentrations were determined with a BNP-32 EIA kit according to the manufacturer's instructions (Phoenix Pharmaceuticals Inc, Burlingame, CA, USA), and IL- $1 \beta$ concentrations were determined with a Rat IL- $1 \beta$ ELISA kit according to the manufacturer's instructions (Pierce Biotechnology Inc, Rockford, IL, USA). Absorbance at $450 \mathrm{~nm}$ was measured, and concentrations determined by interpolation from a standard calibration curve.

\section{Immunohistochemistry}

Tissue samples were fixed at room temperature in $10 \%$ formalin. Samples from human hearts and the hearts of normal rats and those with EAM on day 17 were sequentially dehydrated through an alcohol series and embedded in paraffin. Sections $4-\mu \mathrm{m}$ thick were cut, deparaffinized in xylene, and dehydrated in descending dilutions of ethanol. Specimens were treated by incubating them in EDTA $(\mathrm{pH}$ 8.0) buffer at $121^{\circ} \mathrm{C}$ for $15 \mathrm{~min}$ in an autoclave. After washing in $0.01 \mathrm{~mol} / \mathrm{L}$ phosphate-buffered saline (PBS), endogenous peroxidase activity was blocked by treatment for $20 \mathrm{~min}$ with $0.3 \%$ hydrogen peroxidase in absolute methanol. Specimens were incubated with $10 \%$ normal goat serum for $10 \mathrm{~min}$ at room temperature and for both rats and humans they were reacted with rabbit anti-Lcn2/NGAL antibody (abcam, Cambridge, UK) overnight at $4^{\circ} \mathrm{C}$. The samples were then incubated for $2 \mathrm{~h}$ at room temperature with appropriate second- 

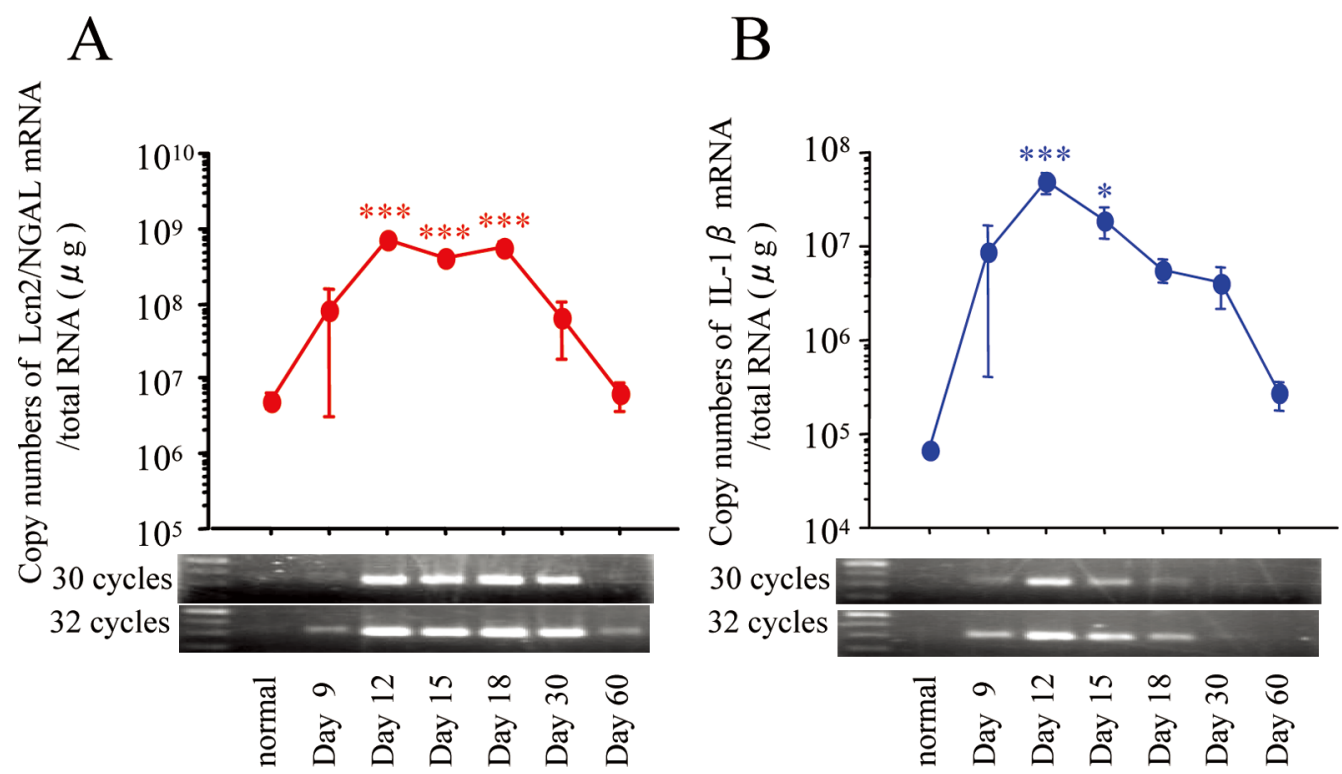

Figure 1. Time course of gene expression of $\operatorname{Lcn} 2 / \operatorname{NGAL}(\mathbf{A})$ and $I L-1 \beta(B)$ in normal $(n=3)$ and EAM hearts on day $9,12,15,18$, 30 ( $n=3$ for each), and $60(n=4)$. PCR bands after 30 and 32 cycles were visualized by ethidium bromide. Error bars represent SEM. Statistical assessment was performed by 1-way ANOVA and Bonferroni's multiple comparison test. Significant differences between normal (8-week old rats without adjuvant injection) and other groups are shown. Differences were considered significant at $P<0.05$. ${ }^{* *} P<0.001$ vs normal rat; ${ }^{* *} P<0.01$ vs normal rat; ${ }^{*} P<0.05$ vs normal rat. EAM, experimental autoimmune myocarditis; IL-1, interleukin-1; Lcn2, lipocalin-2; NGAL, neutrophil gelatinase-B associated lipocalin; PCR, polymerase chain reaction.

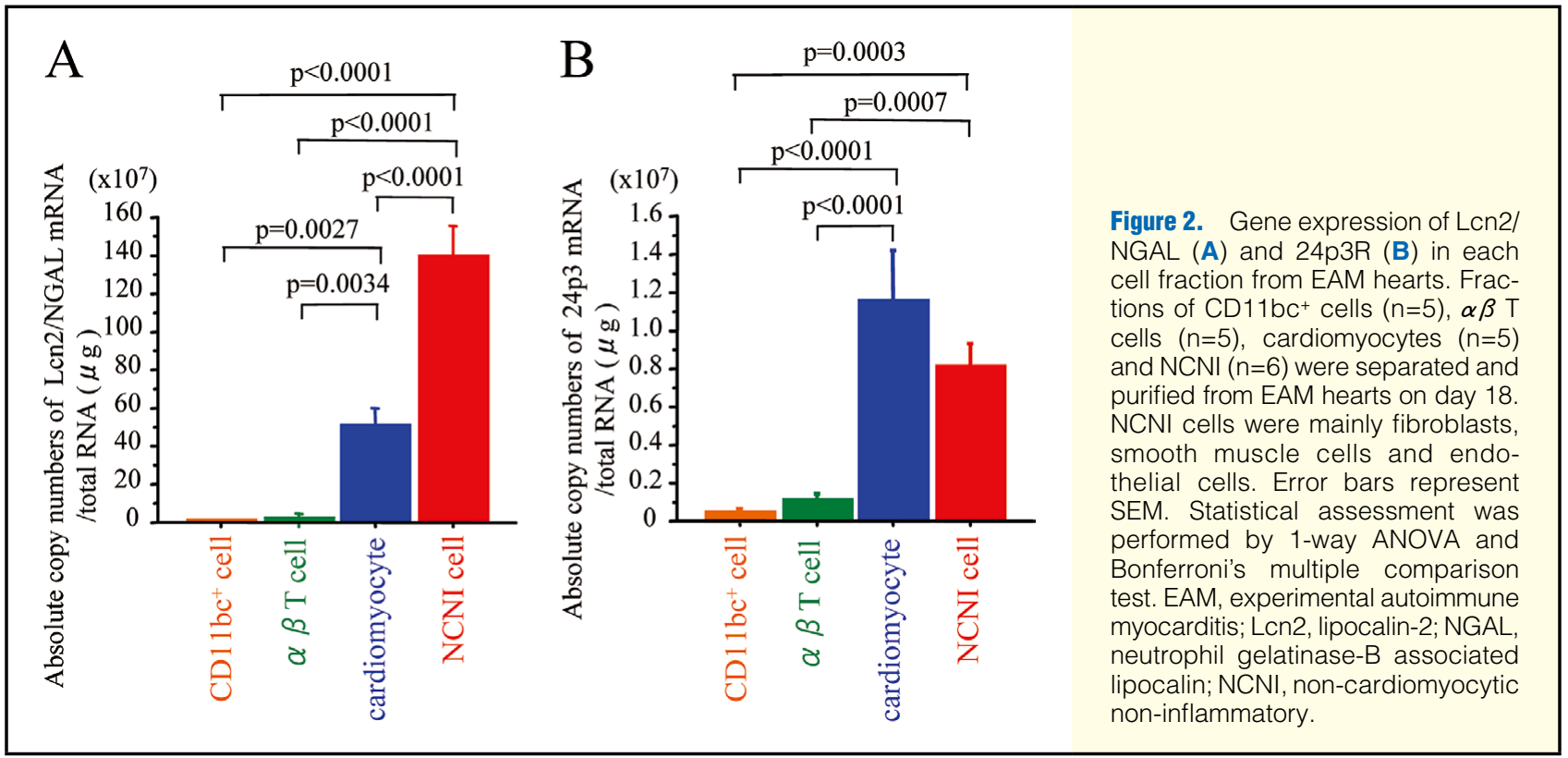

ary antibody (Nichirei, Tokyo, Japan). After this the specimens were carefully washed 3 times with PBS between each step of the procedure. Finally, they were visualized with

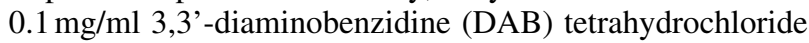
(Dojin Chemical, Kumamoto, Japan), and counterstained with Mayer's hematoxylin.

\section{Electrophoresis and Western Blot Analysis}

Lysates from autopsied human hearts were prepared, and equal amounts $(40 \mu \mathrm{g})$ of denatured proteins were loaded and separated on $16.5 \%$ SDS-PAGE (Mini Protean II; Biorad), and transferred to polyvinylidene difluoride membranes. The membranes were blocked with $10 \%$ dry milk in Tris-buffered saline (TBS) for $1 \mathrm{~h}$. Lcn2/NGAL protein was determined by incubation with 1:1,000 rat anti-human Lcn2/NGAL antibody (R\&D Systems Inc, Minneapolis, MN, USA) overnight in TBS at $4{ }^{\circ} \mathrm{C}$. Membranes were washed 3 times in TBS with $0.05 \%$ Triton $\mathrm{X}-100$, followed by incubation for $1 \mathrm{~h}$ with 


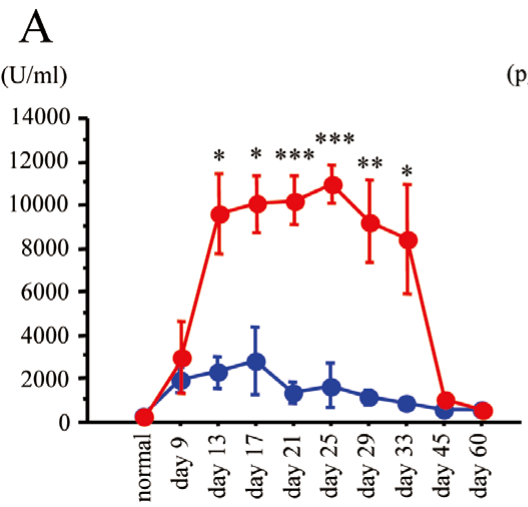

B

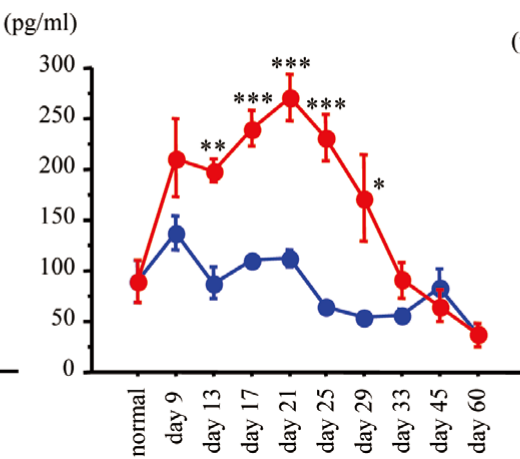

$\mathrm{C}$

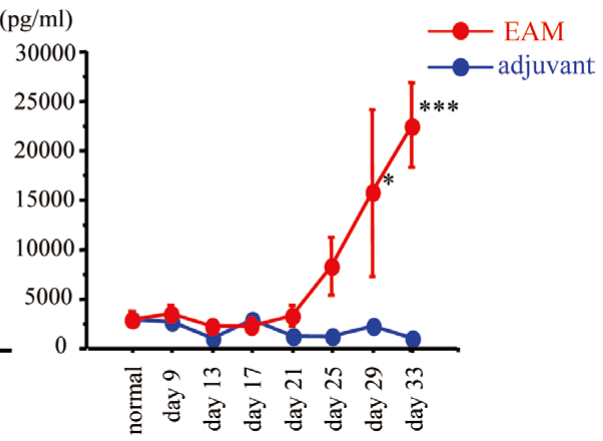

Figure 3. Plasma levels of Lcn2/NGAL (A), IL-1 $\beta(B)$ and BNP (C) measured by ELISA in normal, adjuvant control, and EAM rats $(n=4$ for each). Statistical assessment was performed by 1-way ANOVA and Bonferroni's multiple comparison test. Differences were considered significant at $P<0.05$. ${ }^{* *} P<0.001$ vs normal rat; ${ }^{* *} P<0.01$ vs normal rat; ${ }^{*} P<0.05$ vs normal rat. EAM, experimental autoimmune myocarditis; IL-1, interleukin-1; Lcn2, lipocalin-2; NGAL, neutrophil gelatinase-B associated lipocalin; BNP, B-type natriuretic peptide.

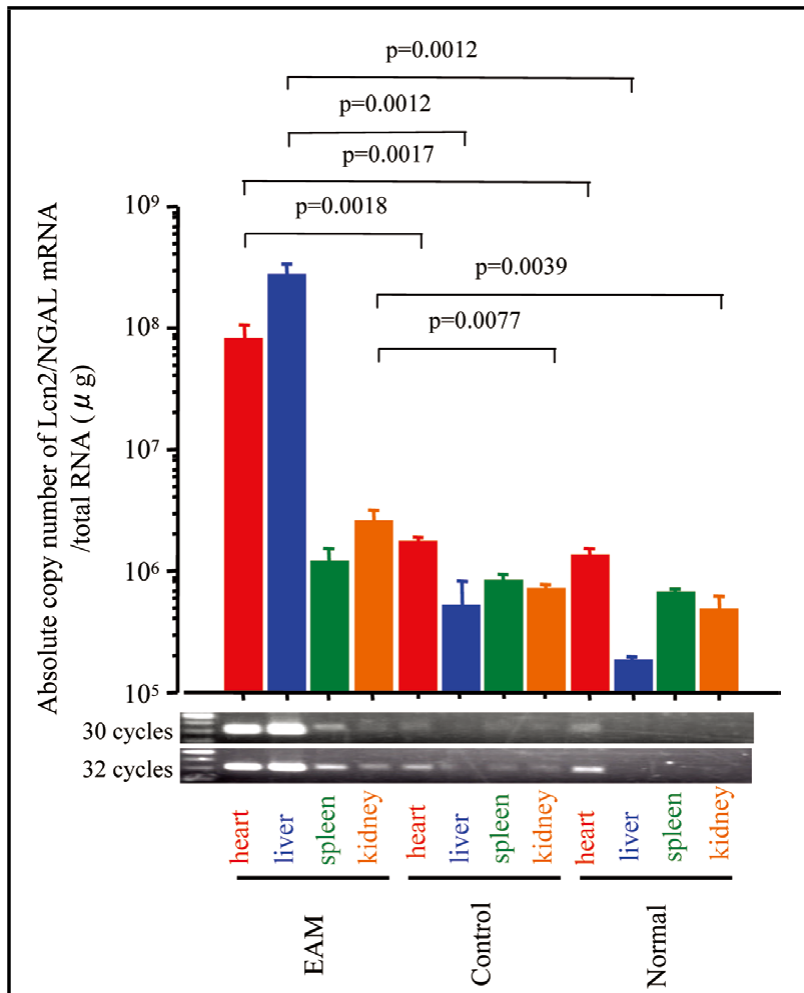

Figure 4. Gene expression of Lcn2/NGAL mRNA in organs of EAM and control rats killed on day 13, and in normal rats ( $n=4$ in each group). Portions of their hearts, livers, spleens, and kidneys were collected. PCR bands after 30 and 32 cycles were visualized by ethidium bromide. Statistical assessment was performed by one-way ANOVA and Bonferroni's multiple comparison test. Differences were considered significant at $\mathrm{P}<0.05$. EAM, experimental autoimmune myocarditis; IL-1, interleukin-1; Lcn2, lipocalin-2; NGAL, neutrophil gelatinase-B associated lipocalin; PCR, polymerase chain reaction. horseradish peroxidase-labeled anti-rat antibody (Santa Cruz Biotechnology, Santa Cruz, CA, USA) in TBS with $0.05 \%$ Triton X-100. The membranes were exposed to a chemiluminescent reagent (GE Healthcare, Buckingham, UK) and autoradiographed for $10 \mathrm{~s}$.

\section{Heart Samples From Heart Failure Patients}

Four autopsied human hearts from individuals who had died of cardiac failure (Case 1: 20-year-old woman diagnosed as having primary pulmonary hypertension; Case 2: 66-yearold man with fulminant myocarditis diagnosed at autopsy; Case 3: 64-year-old man diagnosed at autopsy as having chronic myocarditis; Case 4: 62-year-old man with fulminant myocarditis diagnosed at autopsy) were evaluated by immunohistochemistry. Samples collected from hearts of 26 previously reported patients ${ }^{21}$ (19 patients without myocarditis, 7 patients with myocarditis) were examined for the expression of Lcn2/NGAL by real-time RT-PCR analysis. Lcn2/NGAL in the hearts of Cases 1,2 and 3 were analyzed by Western blot analysis.

The local ethics committee approved this study, and families of all patients signed informed consent in relation to diagnosis by histological examination, including gene expression analyses.

\section{Statistical Analysis}

Statistical assessment was performed by 1-way ANOVA and Bonferroni's multiple comparison test or the Mann-Whitney $\mathrm{U}$ test. Differences were considered significant at $\mathrm{P}<0.05$. Data obtained from quantitative RT-PCR were expressed as mean \pm SEM.

\section{Results}

\section{Time Course of Lcn2/NGAL and IL-1 $\beta$ Expression in EAM Hearts}

A previous study reported that inflammatory cells begin to infiltrate the heart of EAM rats at approximately day 12, peak at approximately days 14-21, and disappear after day 21, followed by progressive fibrosis. ${ }^{22}$ In our study, Lcn2/ NGAL expression in EAM hearts started to rise at day 9 and 

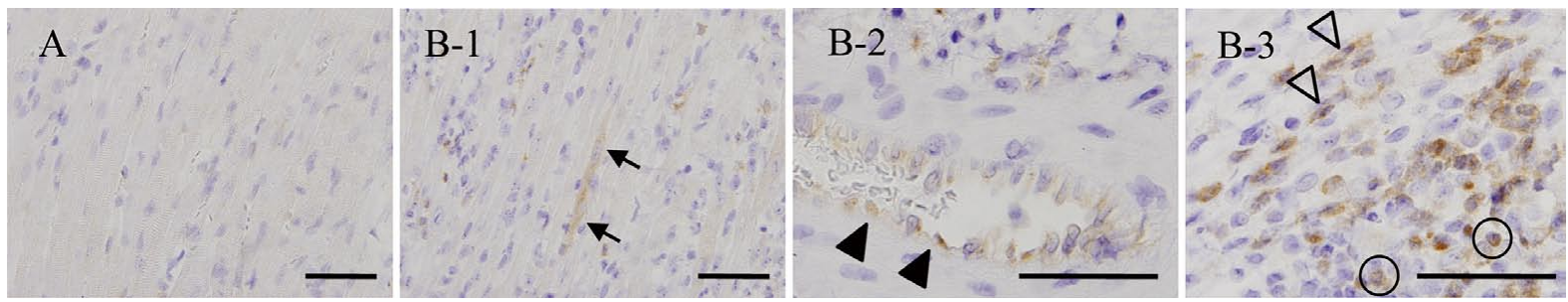

Figure 5. Immunohistochemistry for Lcn2/NGAL in the hearts of a normal rat (A) and an EAM rat on day 17 (B-1-3). Lcn2/NGAL positive cardiomyocytes (arrows), vascular wall cells (arrowhead), spindle-like fibroblastoid cells (white arrowhead) and leukocytes (circle) were detected in hearts of EAM rat. Bars $=100 \mu \mathrm{m}$. EAM, experimental autoimmune myocarditis; Lcn2, lipocalin-2; NGAL, neutrophil gelatinase-B associated lipocalin.

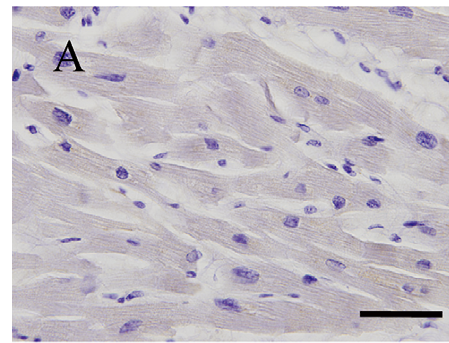

Case 1

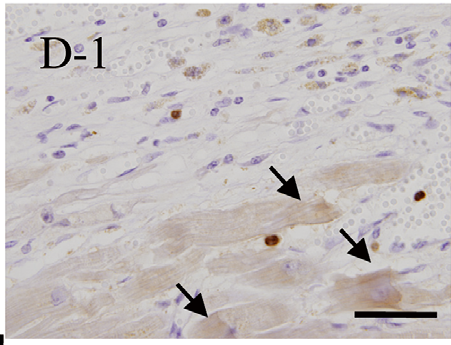

$\mathrm{E}$

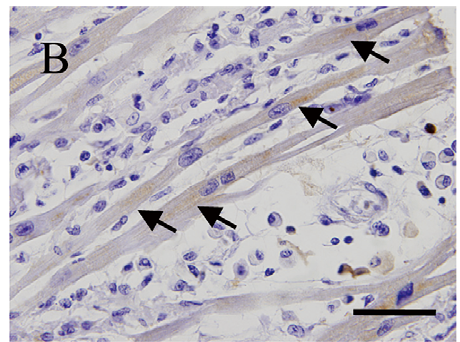

Case 2

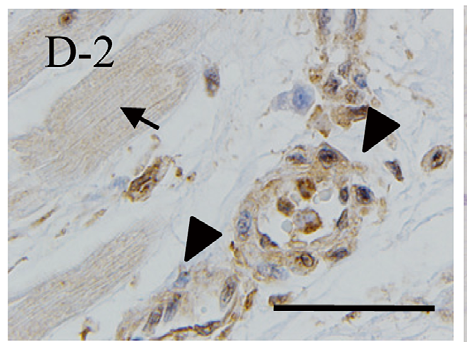

Case 4

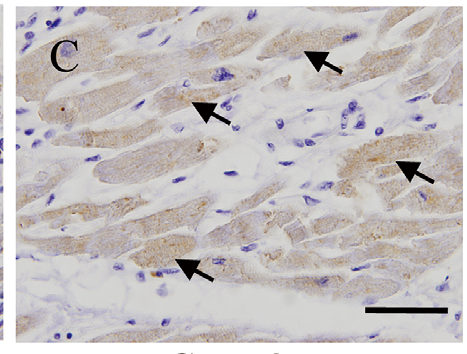

Case 3

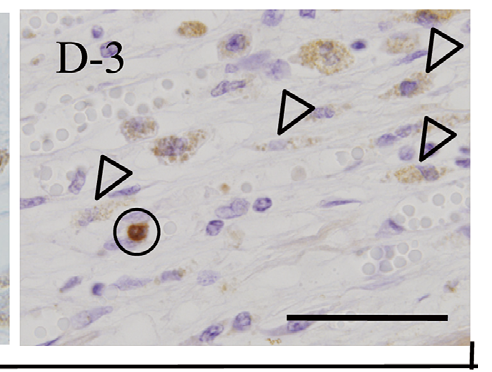

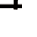 \\ .}

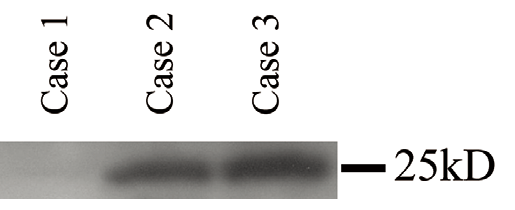

Figure 6. Lcn2/NGAL expression in human hearts. (A-D) Immunohistochemistry for Lcn2/NGAL in hearts of patients with primary pulmonary hypertension (Case 1, A), fulminant myocarditis (Case 2, B), chronic myocarditis (Case 3, C) and fulminant myocarditis (Case 4, D-1, D-2 and D-3). Lcn2/NGAL positive cardiomyocytes (arrows), vascular wall cells (arrowhead), spindle-like fibroblastoid cells (white arrowhead) and leukocytes (circle) were readily detected in hearts of patients with myocarditis. Bars $=100 \mu \mathrm{m}$. (E) Lcn2/NGAL protein levels by Western blot analysis in the hearts of Cases 1-3. Lcn2, lipocalin-2; NGAL, neutrophil gelatinase-B associated lipocalin.

increased approximately 100 -fold on days 12,15 and 18 compared with normal hearts of 8 -week old rats. It then decreased at day 30 and returned to near normal levels at day 60 (Figure 1A). IL-1 $\beta$ expression in the EAM hearts also began to increase at day 9 and increased approximately 700fold at day 12 and then slowly decreased and returned to near normal levels at day 60 (Figure 1B).

\section{Analysis of Lcn2/NGAL or 24p3R mRNA-Expressing Cells in EAM Hearts}

Lcn2/NGAL expressing cells in EAM hearts at day 18 were identified as cardiomyocytes and NCNI cells such as endo- 


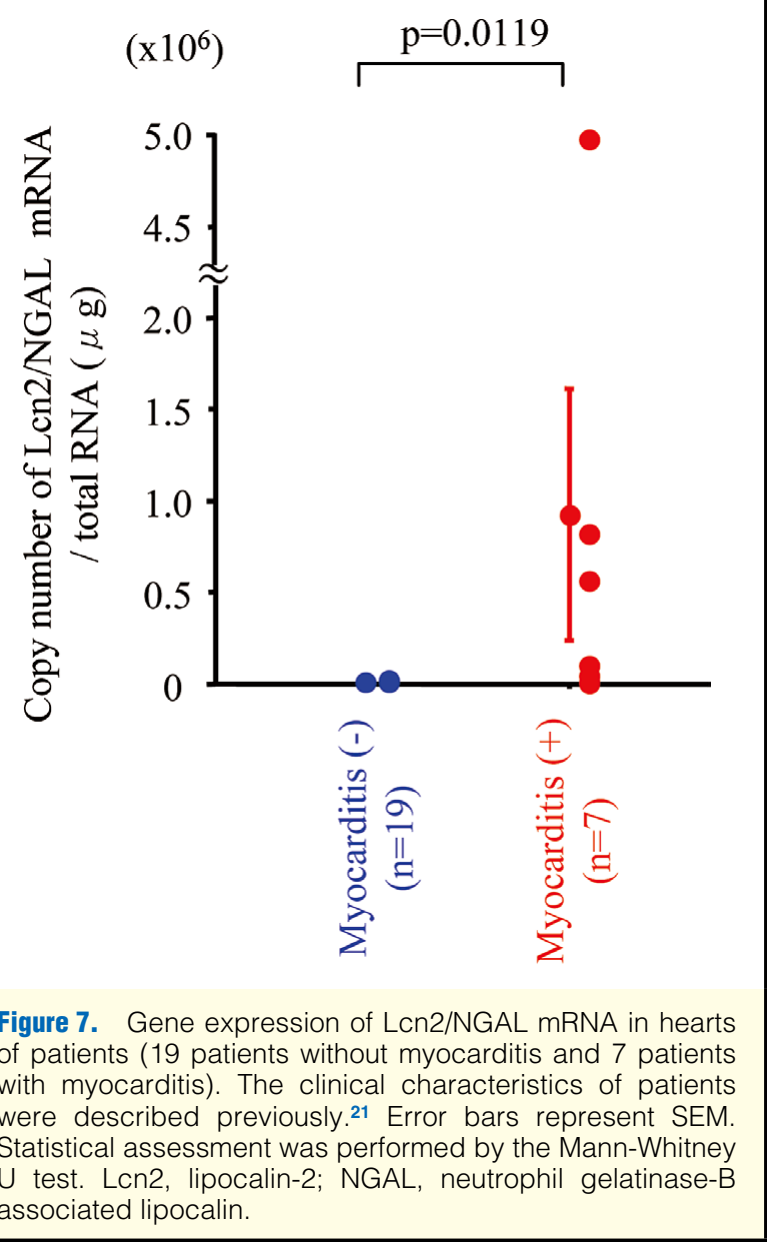

thelial cells, pericytes, smooth muscle cells and fibroblasts (Figure 2A). Lcn2/NGAL specific receptor (24p3R) expressing cells were also identified as cardiomyocytes and NCNI cells (Figure 2B).

\section{Time Course of Plasma Len2/NGAL, IL-1 $\beta$, and BNP Levels in EAM}

Plasma Lcn2/NGAL levels in EAM rats were significantly increased at day 13 compared with normal rats and control rats injected with adjuvant alone, and the high levels of plasma Lcn2/NGAL continued until day 33 but decreased to the near-normal range after day 45 (Figure 3A). The time course of plasma Lcn2/NGAL was similar to that of $\mathrm{Lcn} 2 /$ NGAL expression in EAM hearts. Plasma IL- $1 \beta$ levels in EAM rats showed an increase at day 9 and were significantly higher than in control rats from day 13 to day 29, but decreased thereafter (Figure 3B). As previously reported for IL- $1 \beta$-induced Lcn $2 / \mathrm{NGAL}$ expression, the time course of plasma Lcn2/NGAL was also similar to that of IL-1 $\beta$. On the other hand, plasma BNP levels in the EAM rats slowly increased after the peak of infiltration by inflammatory cells compared with control rats (Figure 3C) and the time course of plasma BNP differed from that of Len2/NGAL.

\section{Analysis of Lcn2/NGAL and IL-1 $\beta$-Expressing Organs in EAM}

Because Lcn2/NGAL expression and plasma Lcn2/NGAL levels in EAM hearts increased significantly on days 12 and
13, respectively, we investigated Lcn2/NGAL expression in the livers, kidneys and spleens of EAM rats at day 13. Lcn2/ NGAL mRNA was significantly increased not only in the hearts but also in the livers. Lcn2/NGAL mRNA expression in the kidneys was also increased, but to a lesser degree than in the hearts or livers (Figure 4).

\section{Lcn2/NGAL Immunostaining in Rat and Human Hearts}

Lcn2/NGAL immunostaining was hardly detected in normal rat hearts (Figure 5A). In the EAM rat hearts, on the other hand, it was found in cardiomyocytes (Figure 5B-1), vascular wall cells (Figure 5B-2), spindle-like fibroblastoid cells and leukocytes (Figure 5B-3). In the heart of Case 1 with primary pulmonary hypertension, Lcn $2 / \mathrm{NGAL}$ immunostaining was not detected (Figure 6A); however, in the hearts of Cases 2-4 with myocarditis, it was found in cardiomyocytes (Figures 6B, C, D-1,D-2), vascular wall cells (Figure 6D-2), spindle-like fibroblastoid cells and leukocytes (Figure 6D-3).

\section{Western Blot Analysis of Lcn2/NGAL in Human Hearts}

In the heart of Case 1 with primary pulmonary hypertension, Lcn2/NGAL protein in the heart was hardly detected by Western blot analysis, but was detected in the hearts of Cases 2 and 3 (Figure 6E).

\section{Lcn2/NGAL mRNA Expression in Human Hearts}

Lcn2/NGAL mRNA levels in human hearts with myocarditis measured by real-time RT-PCR were significantly greater than in those without myocarditis (Figure 7). However, as shown in our recent studies, ${ }^{21}$ there was no significant difference in BNP mRNA levels.

\section{Discussion}

Recently Yndestad et al ${ }^{14}$ reported that Lcn $2 /$ NGAL is highly expressed in the cardiomyocytes of heart failure patients, and might be a useful biomarker of the severity of heart failure. In the current study we demonstrated that Lcn2/NGAL expression in the hearts of rats and humans with myocarditis, as well as the plasma Lcn2/NGAL levels in EAM rats, were significantly increased compared with normal or control rats. However the increase in Lcn2/NGAL in our study was more pronounced than in the study of Yndestad et al. ${ }^{14}$ Moreover, our time course analysis in EAM rats showed that elevation of both the cardiac expression of Lcn2/NGAL and of the plasma Lcn2/NGAL level was more marked during the active stages of myocarditis, and closely paralleled cardiac IL- $1 \beta$ expression levels and plasma IL- $1 \beta$ levels. Yndestad et al demonstrated that IL-1-induced Lcn2/NGAL expression occurs in rat neonatal cardiomyocytes, and we made similar observations (data not shown). Interleukins like IL-1 usually act in an autocrine/paracrine fashion, not in an endocrine fashion. Collectively these observations suggest that Lcn2/NGAL expression in hearts is markedly increased, in synchrony with the timing of elevation of cytokines such as IL-1 in the heart.

$\mathrm{Lcn} 2 / \mathrm{NGAL}$ is a novel protein involved in iron transport. ${ }^{4}$ In the current study, its specific receptor (24p3R) was also expressed in cardiomyocytes and NCNI cells. We therefore hypothesized that Lcn2/NGAL acts in an autocrine fashion. Cardiomyocytes possess considerable amounts of protein, including an abundance of iron-containing myoglobin and ferritin. ${ }^{23,24}$ When cardiac injury occurs, iron is thought to be dispersed within the extracellular space of the heart. 
Although iron is an essential nutrient in all cells, ${ }^{25}$ enhanced oxidative stress because of excessive iron may cause lethal damage to cells. ${ }^{26}$ In a recent study we demonstrated that expression of hepcidin, another novel protein involved in iron transport, was increased in cardiomyocytes in myocarditis or acute myocardial infarction. ${ }^{21}$ In the presence of massive cardiac injury, proteins that are involved in iron transport may play an important role in iron homeostasis. We speculate that they may act by reducing extracellular iron concentrations in the heart, perhaps through the mechanism of sequestering, storing, and detoxification in the form of ferritin. ${ }^{27}$ Thus they may play an important cytoprotective role against extracellular free radical formation by inhibiting an increase in the extracellular iron concentration. It has also been reported that $\mathrm{Lcn} 2 / \mathrm{NGAL}$ prevents $\mathrm{H}_{2} \mathrm{O}_{2}$ toxicity, which is considered to be an inducer of oxidative stress caused by reactive oxygen species generation, thus providing a potential beneficial effect in ameliorating the toxicity induced by oxidative stress conditions. ${ }^{5}$ However, further studies are needed to elucidate in full the functions of Lcn2/ NGAL in cardiac injury.

Recently several studies have shown that plasma and urine Lcn2/NGAL levels might be useful biomarkers of acute renal failure, ${ }^{8,9,11,28,29}$ and it has been suggested that proximal tubules produce Lcn2/NGAL in acute renal failure. ${ }^{30,31}$ In our current study we demonstrated that expression of Lcn2/ NGAL was greatest in hearts and livers. Although it remains unknown why the livers in EAM rats express Lcn2/NGAL so prominently, we speculate that this may be caused by high plasma IL-1 levels or liver congestion because of heart failure. In any case, if multiple organ failure occurs, high plasma Lcn2/NGAL levels should be carefully evaluated, considering that several organs can express Lcn2/NGAL. Moreover, in the presence of dramatic changes such as occur in myocarditis, plasma Lcn2/NGAL levels should also be evaluated with respect to the stage of disease. Biomarkers that predict preexisting heart disease and the severity of cardiac remodeling are greatly needed. ${ }^{32-34}$ The present study suggests that Lcn2/NGAL is strongly expressed and induced by proinflammatory cytokines in hearts with myocarditis and we speculate that it may play a cytoprotective role by transporting iron into cells and thus may be a useful biomarker of inflammatory heart disease, but further studies are needed to evaluate the full potential of Lcn2/NGAL.

\section{Acknowledgments}

This study was supported in part by a Ministry of Health, Labor and Welfare in Japan Grant "Research on Regulatory Science of Pharmaceutical and Medical Devices." and by a Grant for scientific research from the Ministry of Education, Culture, Sports, Science and Technology of Japan (no. 20591185).

\section{References}

1. Kjeldsen L, Johnsen AH, Sengelov H, Borregaard N. Isolation and primary structure of NGAL, a novel protein associated with human neutrophil gelatinase. J Biol Chem 1993; 268: 10425-10432.

2. Flo TH, Smith KD, Sato S, Rodriguez DJ, Holmes MA, Strong RK, et al. Lipocalin 2 mediates an innate immune response to bacterial infection by sequestrating iron. Nature 2004; 432: 917-921.

3. Devireddy LR, Gazin C, Zhu X, Green MR. A cell-surface receptor for lipocalin $24 \mathrm{p} 3$ selectively mediates apoptosis and iron uptake. Cell 2005; 123: $1293-1305$.

4. Richardson DR. 24p3 and its receptor: Dawn of a new iron age? Cell 2005; 123: 1175-1177.

5. Roudkenar MH, Halabian R, Ghasemipour Z, Roushandeh AM, Rouhbakhsh M, Nekogoftar M, et al. Neutrophil gelatinase-associated lipocalin acts as a protective factor against $\mathrm{H}(2) \mathrm{O}(2)$ toxicity. Arch Med Res 2008; 39: 560-566.
6. Cowland JB, Sorensen OE, Sehested M, Borregaard N. Neutrophil gelatinase-associated lipocalin is up-regulated in human epithelial cells by IL-1 beta, but not by TNF-alpha. J Immunol 2003; 171: 6630-6639.

7. Schmidt-Ott KM, Mori K, Li JY, Kalandadze A, Cohen DJ, Devarajan P, et al. Dual action of neutrophil gelatinase-associated lipocalin. J Am Soc Nephrol 2007; 18: 407-413.

8. Mishra J, Dent C, Tarabishi R, Mitsnefes MM, Ma Q, Kelly C, et al. Neutrophil gelatinase-associated lipocalin (NGAL) as a biomarker for acute renal injury after cardiac surgery. Lancet 2005; 365: $1231-1238$

9. Poniatowski B, Malyszko J, Bachorzewska-Gajewska H, Malyszko JS, Dobrzycki S. Serum neutrophil gelatinase-associated lipocalin as a marker of renal function in patients with chronic heart failure and coronary artery disease. Kidney Blood Press Res 2009; 32: $77-80$.

10. Hirsch R, Dent C, Pfriem H, Allen J, Beekman RH 3rd, Ma Q, et al. NGAL is an early predictive biomarker of contrast-induced nephropathy in children. Pediatr Nephrol 2007; 22: 2089-2095.

11. Damman K, van Veldhuisen DJ, Navis G, Voors AA, Hillege HL. Urinary neutrophil gelatinase associated lipocalin (NGAL), a marker of tubular damage, is increased in patients with chronic heart failure. Eur J Heart Fail 2008; 10: 997-1000.

12. Aigner F, Maier HT, Schwelberger HG, Wallnofer EA, Amberger $\mathrm{A}$, Obrist $\mathrm{P}$, et al. Lipocalin-2 regulates the inflammatory response during ischemia and reperfusion of the transplanted heart. Am J Transplant 2007; 7: 779-788.

13. Roudkenar MH, Kuwahara Y, Baba T, Roushandeh AM, Ebishima $\mathrm{S}$, Abe S, et al. Oxidative stress induced lipocalin 2 gene expression: Addressing its expression under the harmful conditions. J Radiat Res (Tokyo) 2007; 48: 39-44.

14. Yndestad A, Landro L, Ueland T, Dahl CP, Flo TH, Vinge LE, et al. Increased systemic and myocardial expression of neutrophil gelatinase-associated lipocalin in clinical and experimental heart failure. Eur Heart J 2009; 30: 1229-1236.

15. Watanabe R, Hanawa H, Yoshida T, Ito M, Isoda M, Chang $\mathrm{H}$, et al. Gene expression profiles of cardiomyocytes in rat autoimmune myocarditis by DNA microarray and increase of regenerating gene family. Transl Res 2008; 152: 119-127.

16. Liu H, Hanawa H, Yoshida T, Elnaggar R, Hayashi M, Watanabe $\mathrm{R}$, et al. Effect of hydrodynamics-based gene delivery of plasmid DNA encoding interleukin-1 receptor antagonist-Ig for treatment of rat autoimmune myocarditis: Possible mechanism for lymphocytes and noncardiac cells. Circulation 2005; 111: $1593-1600$.

17. Chang H, Hanawa H, Yoshida T, Hayashi M, Liu H, Ding L, et al. Alteration of IL-17 related protein expressions in experimental autoimmune myocarditis and inhibition of IL-17 by IL-10-Ig fusion gene transfer. Circ J 2008; 72: 813-819.

18. Lane JR, Neumann DA, Lafond-Walker A, Herskowitz A, Rose NR. Role of IL-1 and tumor necrosis factor in coxsackie virusinduced autoimmune myocarditis. J Immunol 1993; 151: 16821690.

19. Loppnow H, Westphal E, Buchhorn R, Wessel A, Werdan K. Interleukin-1 and related proteins in cardiovascular disease in adults and children. Shock 2001; 16: 3-9.

20. Yoshida T, Hanawa H, Toba K, Watanabe H, Watanabe R, Yoshida $\mathrm{K}$, et al. Expression of immunological molecules by cardiomyocytes and inflammatory and interstitial cells in rat autoimmune myocarditis. Cardiovasc Res 2005; 68: 278-288.

21. Isoda M, Hanawa H, Watanabe R, Yoshida T, Toba K, Yoshida K, et al. Expression of the peptide hormone hepcidin increases in cardiomyocytes under myocarditis and myocardial infarction. J Nutr Biochem 2009 [E-pub ahead of print].

22. Kodama M, Matsumoto Y, Fujiwara M, Masani F, Izumi T, Shibata A. A novel experimental model of giant cell myocarditis induced in rats by immunization with cardiac myosin fraction. Clin Immunol Immunopathol 1990; 57: 250-262.

23. Walters FP, Kennedy FG, Jones DP. Oxidation of myoglobin in isolated adult rat cardiac myocytes by 15 -hydroperoxy-5,8,11,13eicosatetraenoic acid. FEBS Lett 1983; 163: 292-296.

24. Baron CP, Andersen HJ. Myoglobin-induced lipid oxidation: A review. J Agric Food Chem 2002; 50: 3887-3897.

25. Aisen P, Enns C, Wessling-Resnick M. Chemistry and biology of eukaryotic iron metabolism. Int J Biochem Cell Biol 2001; 33: 940-959.

26. Halliwell B, Gutteridge JM. Biologically relevant metal ion-dependent hydroxyl radical generation: An update. FEBS Lett 1992; 307: $108-112$.

27. Balla G, Jacob HS, Balla J, Rosenberg M, Nath K, Apple F, et al. Ferritin: A cytoprotective antioxidant strategem of endothelium. 
J Biol Chem 1992; 267: 18148-18153.

28. Bolignano D, Donato V, Coppolino G, Campo S, Buemi A, Lacquaniti A, et al. Neutrophil gelatinase-associated lipocalin (NGAL) as a marker of kidney damage. Am J Kidney Dis 2008; 52: 595-605.

29. Bolignano D, Basile G, Parisi P, Coppolino G, Nicocia G, Buemi $M$. Increased plasma neutrophil gelatinase-associated lipocalin levels predict mortality in elderly patients with chronic heart failure. Rejuvenation Res 2009; 12: 7-14.

30. Schmidt-Ott KM, Mori K, Kalandadze A, Li JY, Paragas N, Nicholas T, et al. Neutrophil gelatinase-associated lipocalin-mediated iron traffic in kidney epithelia. Curr Opin Nephrol Hypertens 2006; 15: 442-449.

31. Mishra J, Ma Q, Prada A, Mitsnefes M, Zahedi K, Yang J, et al.
Identification of neutrophil gelatinase-associated lipocalin as a novel early urinary biomarker for ischemic renal injury. $J$ Am Soc Nephrol 2003; 14: 2534-2543.

32. Saiki A, Iwase M, Takeichi $Y$, Umeda H, Ishiki R, Inagaki $H$, et al. Diversity of the elevation of serum cardiac troponin I levels in patients during their first visit to the emergency room. Circ J 2007; 71: $1458-1462$.

33. Terasaki F, Okamoto H, Onishi K, Sato A, Shimomura H, Tsukada $B$, et al. Higher serum tenascin-C levels reflect the severity of heart failure, left ventricular dysfunction and remodeling in patients with dilated cardiomyopathy. Circ J 2007; 71: 327-330.

34. Okamoto H. Can adiponectin be a novel metabolic biomarker for heart failure? Circ J 2009; 73: 1012-1013. 